\title{
Telomerase promoter reprogramming and interaction with general transcription factors in the human mesenchymal stem cell
}

\author{
Nedime Serakinci ${ }^{1}$, \\ Stacey F H oare?, \\ M oustapha Kassem ${ }^{3}$, \\ Stuart P Atkinson ${ }^{2} \&$ \\ W Nicol Keith ${ }^{2 \dagger}$ \\ ${ }^{\dagger}$ Author for correspondence \\ 1 U niversity of Southern \\ D enmark, Institute of \\ M edical Biology, D epartment \\ of Anatomy and \\ N eurobiology, D K-5000 \\ 0 dense $C, D$ enmark \\ 2U niversity of Glasgow, \\ Centre for 0 ncology and \\ Applied Pharmacology, \\ Cancer Research U K Beatson \\ Laboratories, Garscube \\ Estate, Switchback Road, \\ Bearsden, \\ Glasgow G61 1BD, UK \\ Tel.: +44 141330 4811; \\ Fax: +44 141330 4127; \\ E-mail: n.keith@ \\ beatson.gla.ac.uk \\ $3 \mathrm{U}$ niversity $\mathrm{H}$ ospital of \\ $O$ dense, $D$ epartment of \\ Endocrinology and \\ M etabolism, D K-5000 \\ 0 dense $C, D$ enmark
}

Keywords chromatin epigenetics, hTERT, hTR, mesenchymal, telomerase, stem cell

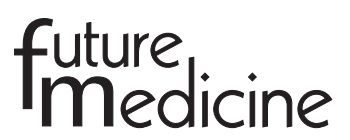

\begin{abstract}
The human adult mesenchymal stem cell (hMSC) does not express telomerase and has been shown to be the target for neoplastic transformation after transduction with hTERT. These findings lend support to the stem cell hypothesis of cancer development but by supplying hTERT, the molecular events required to upregulate hTERT expression in cancer development are missed. Therefore, the hMSC is ideal for the identification of molecular mechanisms regulating telomerase gene expression in stem cells. This study shows that the repression of hTERT expression in hMSC is chromatin based and that modifications of the chromatin environment lead to reactivation of telomerase gene expression. It is shown that repression of hTERT expression in hMSCs is due to promoter-specific histone hypoacetylation coupled with low Pol II and TFIIB trafficking. This repression is overcome by treatment with Trichostatin A (TSA), an HDAC inhibitor, concomitant with increases in promoter-specific histone acetylation and increases in Pol II and TFIIB tracking. hTR expression is also increased in TSA-treated hMSCS, concomitant with changes in Pol II and TFIIB dynamics.
\end{abstract}

Stem cells are unique in their ability to both self renew and to give rise to differentiated tissues. M esenchymal stem cells (hM SC) are multipotential cells. Thus, hM SC have the ability both in vivo and in vitro, to differentiate into a variety of adult mesenchymal tissues, such as bone, cartilage, adipose and muscle [1,2] and therefore may be of use in the treatment of a diverse variety of clinical conditions. In addition, there are now strong links between normal stem cells and cancer stem cells [3], suggesting that stem cells are targets for neoplastic transformation. Interestingly, despite having stem cell properties, when passaged in culture the hM SC gradually reduce their proliferative rate and enter replicative senescence $[1,2]$. hM SC 5 also lack activity for the immortalizing enzyme telomerase which can counteract senescence by maintaining telomere sequence [1,2]. Further, when hTERT is ectopically expressed in the hM SC, neoplastic changes are accumulated suggesting that the adult hMSCs can be targets for neoplastic transformation [4].

The expression of telomerase is regulated in normal human cells. During development, telomerase expression is extinguished after embryonic differentiation in most cells. N otable exceptions are male germ cells, lymphocytes and some stem cell populations, which maintain their proliferative capacity in adult life, are telomerase competent and can express low levels of telomerase. By contrast, telomerase is highly active in the vast majority of human tumors [5-7]. Thus, a key issue is how this complex pattern of expression is maintained in adult tissues and in particular the adult stem cell and how this is deregulated during neoplastic progression [8].

Telomerase activity is achieved through the expression of two genes, the hTR gene, which codes for the essential RNA component of telomerase, and the hTERT gene, which codes for the telomerase protein component that has reverse transcriptase activity. A major mechanism to differentially regulate telomerase is transcriptional control of the hTERT and hTR genes. Although much remains to be clarified, the cloning of the hTR and hTERT promoter region has enabled the identification of several positive and negative regulators of transcription [9].

H owever, gene expression is not only determined by the availability of combinations of transcription factors but also by chromatin context and in particular by covalent modifications of the core histones including acetylation, phosphorylation and methylation [10]. Distinct modification patterns at promoter sequences have been linked to expression and repression, and the dynamics of histone modification in transcriptional control is ideally suited to the task of organizing complex programs of gene activity necessary for many cellular processes including cell lineage commitment, cellular senescence and tumorigenesis [11-13]. Formation 
of the basal transcriptional machinery into transcriptional complexes on promoter sequences is also a major step in gene transcription. It is known that telomerase has a critical role in tumor progression and cancer cell survival; therefore it is crucial that the mechanisms regulating telomerase gene expression are well understood. Studies have shown that distinct patterns of histone modifications are linked to hTR and hTERT gene regulation [14] and alterations in expression levels are concomitant with dynamic changes in basal transcription machinery binding [15].

Thus, the lack of hTERT expression in the hM SC provides an ideal model to study telomerase gene regulation in the normal adult stem cell. This allows us to study all stages of promoter function including the key steps of transcription preinitiation complex formation, promoter clearance and elongation in the context of chromatin remodeling. The histone deacetylase inhibitor Trichostatin A (TSA) has been shownpreviously to cause an increase in histone acetylation at the hTERT promoter and cause reexpression of the hTERT gene [16-18]. To this end, we have studied hTERT and hTR expression, the acetylation status of the $h T R$ and hTERT promoters and RNA Pol II and TFIIB dynamics in the hMSC by chromatin immunoprecipitation ( $\mathrm{C} / \mathrm{P}$ ), before and after treatment with TSA.

Material \& methods

Isolation of hMSC \& cell culture

Primary hM SC isolated from bone marrow aspirates by centrifugation ( $700 \mathrm{~g}$ for $15 \mathrm{~min}$ at $4^{\circ} \mathrm{C}$ ) over a Ficoll- $\mathrm{H}$ ypaque gradient (Sigma) as previously described by Pittenger and colleagues [19]. The resulting cells were cultivated in high-glucose $(4.5 \mathrm{~g} / \mathrm{l}) \quad$ Dulbecco's modified Eagles medium (DMEM, Gibco, Life Technology, USA) supplemented with $10 \%$ fetal bovine serum (Gibco, Life technology), $100 \mathrm{U} / \mathrm{ml}$ of penicillin and streptomycin (Gibco, Life technology) and $2 \mathrm{mM}$ of L-glutamine. Cells were plated out with a confluence of $75 \%$ and the next day TSA was added at a final concentration of $350 \mu \mathrm{M}$ for $17 \mathrm{~h}$. This was followed by fixing in neutral buffered formalin (at a final concentration of $3.7 \%$ ) for ChIP assay.

Chromatin immunoprecipitation assays ChIP assays for histone acetylation were performed following the instructions recommended by the kit supplier (Upstate
Biotechnology, UK). Antibodies used were anti-acetylated histone $\mathrm{H} 3(\mathrm{AcH} 3)$, which recognizes acetylated lysines 9 and 14 and antiacetylated histone $\mathrm{H} 4(\mathrm{AcH} 4)$, which recognizes acetylated lysines 5, 8, 12 and 16 (U pstate Biotechnology, Cat. N o. 06-599 and 06-866). Sonication was optimized to give chromatin fragments of around $500 \mathrm{bp}$ in length $(8 \times 10$ second pulses at $5 \mu$ with $20 \mathrm{~s}$ rest between each pulse on ice using an M SE Soniprep150 sonifier) and the resultant D N A from each immunoprecipitation (IP) was purified using the QIAquick PCR Purification $\mathrm{Kit}^{\mathrm{TM}}$ (Q iagen, UK). C ChI P assays using antiPol II and anti-TFIIB (both Autogen Bioclear, UK) were undertaken as in Gomez-Ramon and colleagues [20]. In both ChIP techniques and in each individual experiment, a no-antibody control is used to detect any background, which if present is subtracted from the IPs to normalize.

Quantitative polymerase chain reaction Products from the C hIP assay were quantified by quantitative polymerase chain reaction (Q-PCR) O pticon2 D N A Engine (MJ Research, M assachusetts) using primers to hTR and hTERT core promoter sequences and exon 12 of hTERT using SYBR Green Q -PCR Buffer (Finnzymes, Espo0, Finland) [14,15]. hTR Primers, 29SF $5^{\prime} \mathrm{CCC}$ GCC CGA GAG AGT GAC 3 ' and 5ALTR 5' AAG TCA GCG AGA AAA ACA GC 3', hTERT primers, TERTSF 5' TCC CCT TCA CGT CCG GCA TT $3^{\prime}$ and TERTSR $5^{\prime}$ AGC GGA GAG AGG TCG AAT CG $3^{\prime}$ and Exon 12 primers, Ex12Fa 5'CAG GAC AAG GAA GCG GGA GGA 3' Ex 12Rab 5'CAG CCG CAA GAC CCC AAA GA 3' were used.

\section{Expression analysis}

hT R expression was analyzed by Q-PCR using the following primers (TRC3F - 5' CTAACCCTAACTGAGAAGGGCGTA 3 ' and TRC $3 R$ - 5 ' GGCGAACGGGCCAGCAGCTGACAT $T 3^{\prime}$ and adjusted to GAPD H expression (primers - GAPDH0.45F - 5' ACCACAGTCCATGCCATCAC $3^{\prime}$ and GAPDH $0.45 R$ - 5' CCA CCA CCC TGT TGC TGT A 3') [14,15]. hTERT expression was analyzed by methods outlined in Keith and colleagues [21] using primers which detect all splice variants (primers HT2026F $5^{\prime}$ GCCTGAGCTGTACTTTGTCAA 3' AND HT2482R 5' GCCAAACAGCTTGTTCTCCATGTC $\left.3^{\prime}\right)$. hTERT expression is displayed as total amounts of all four main splice variants. 


\section{Chromatin and telomerase gene expression - RESEARCH ARTICLE}

Results

hTERT gene expression in hMSC \& chromatin remodeling at the hTERT promoter

hTERT expression was studied before and after treatment with TSA and levels of histone acetylation at the promoter were also studied by $\mathrm{ChIP}$ assays in order to link changes in the chromatin environment with any changes in gene expression. In control (untreated) hM SCs, there was a lack of expression of any hTERT transcripts (Figre1A). Acetylation of histone $\mathrm{H} 3$ was not detected and acetylation of histone $\mathrm{H} 4$ was low (Figre 1B), linking the lack of expression of hTERT with low levels of promoter proximal histone acetylation. U pon treatment with TSA, hMSCs showed re-expression of full-length hT ERT transcripts (Figre1A) and this was associated with increased levels of $\mathrm{AcH} 3$ and $\mathrm{AcH} 4$, (Figre1B) linking remodeling of the chromatin environment of the hTERT promoter with reexpression of hTERT.

Basal transcription machinery dynamics at the hTERT gene in hMSCS.

As well as monitoring acetylation, Pol II and TFIIB occupancy at the hT ERT promoter and exon 12 of hTERT were also studied, to understand the trafficking of the basal transcription machinery along the hTERT gene. Exon 12 is located some $31 \mathrm{~kb}$ downstream from the promoter and therefore can give excellent data on the extent of basal transcription machinery tracking along the gene-coding sequence. Pol II levels were low at the hT ERT promoter before treatment with TSA and decreased slightly after treatment (Figre 1C) perhaps signifying that any Pol II that was present at the promoter but was not engaged in transcription. Levels of Pol II at exon 12 were increased after treatment with TSA (Figure1C), indicating that increasing amounts of Pol II was involved in the process of transcription of thehTERT gene.

TFIIB levels in hM SC s were increased at the promoter upon TSA treatment, perhaps due to the relaxing of the chromatin environment allowing the stabilization of the preinitiation complex and increased transcription of hTERT by Pol II. Low levels of TFIIB at exon 12 before and after TSA treatment suggests that the role of TFIIB is primarily at the promoter in stabilizing the preinitiation complex (Figre $\mathbf{D D}$ ).

hTR gene expression in hM SC \& chromatin remodeling at the $\mathrm{hTR}$ promoter

hTR gene expression and promoter chromatin environment was also studied in hM SC before and after treatment with TSA. Figure2A shows that hM SCs express hTR and levels increase upon treatment with TSA. H owever, ChIP analysis did not detect any change in acetylation of histones $\mathrm{H} 3$ and $\mathrm{H} 4$ before and after treatment with TSA (Figre2B).

Basal transcription machinery dynamics at the hTR gene in hMSCS

Although there did not seem to be an increase in histone acetylation, hM SC s treated with TSA showed an increase in Pol II and TFIIB levels at the promoter sequence (Figre $2 \mathbf{C}$ ). An increase in Pol II and TFIIB at the promoter could indicate an increase in stability of the transcriptional complex and therefore an increase in transcription levels of hTR that would account for the increase in expression. As the hT R gene is relatively short, extended analysis of Pol II and TFIIB trafficking was not possible.

\section{Discussion}

A common set of features distinguish cancer cells from their normal counterparts including loss of cell cycle regulation, loss of control over invasion and metastasis, failure of apoptotic mechanisms and bypass of senescence $[7,22]$. Cellular models designed to recapitulate these features of multistage neoplastic progression have recently been developed by a number of groups and epitomized by the work from the Weinberg lab [21]. A common element of these models is to use ectopic expression of the hTERT gene to overcome senescence and thus provide the immortalization step of cancer progression. However, whilst this model for neoplastic transformation may be of considerable use in defining a number of the key events in progression, by supplying telomerase, the molecular events required to upregulate hTERT in cancer development are inevitably missed. Given the critical role that telomerase has in tumor progression and with the cancer cell reliant on telomerase for its survival, it is essential that the mechanisms regulating telomerase gene expression are investigated [8]. 0 ur findings give essential information about the regulation of endogenous telomerase gene expression in normal hM SCs. Understanding telomerase gene regulation in the $\mathrm{hMSC}$, and similar cell types, is of importance. Any differences in regulation may reflect distinct regulatory pathways for telomerase gene expression in these cell types, which need not be the same as in normal and cancer cell lines, which has been published previously $[14,15]$. 


\section{Figure 1. hTERT expression, chromatin environment and basal transcriptional machinery dynamics.}
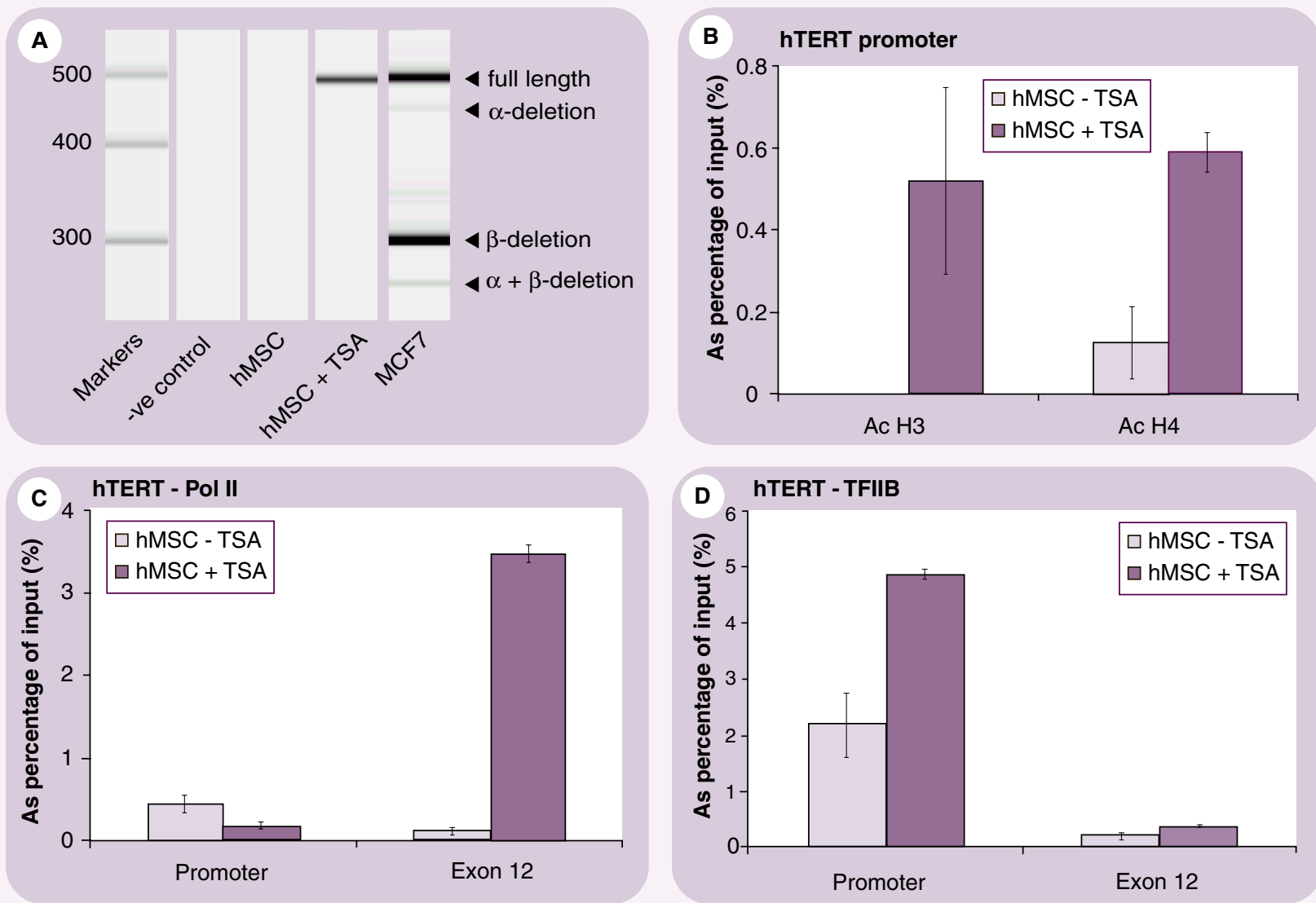

(A) hTERT spice variant expression analysis of hM SC before and after treatment with TSA, corrected to GAPDH expression and visualized using the Agilent Bioanalyser (Agilent Technologies). Lane 1 shows size markers, Lane 2, the negative control for the PCR reaction, Lane 3, hTERT expression for the hM SC, Lane 4, hTERT expression for the hM SC treated with TSA, and Lane 5, hTERT expression in the M CF-7 cell line as comparison. ChIP assays were performed for (B) histone acetylation (histones H3 and H4) status of the hTERT promoter (C) Pol II and (D) TFIIB occupancy of the promoter and exon 12 before and after treatment of hM SCs with TSA. Each IP is expressed as a percentage of an input sample to normalize and detected by Q-PCR in triplicate to generate error bars. No antibody controls were analyzed within each experiment and Q-PCR allowed the subtraction of any background from each IP.

hM SC: Human mesenchymal stem cell; IP: Immunoprecipitation; Q-PCR: Quantitative polymerase chain reaction; TSA: Trichostatin A.

The data presented shows that hTERT expression is repressed in the hM SC and that the mode of repression is chromatin based. Upon TSA treatment of hMSCS, hTERT becomes re-expressed concomitant with an increase in promoter histone acetylation. Beside hTERT expression, hTR expression is also increased upon TSA treatment although large changes in histone acetylation are not apparent. This suggests that chromatin and epigenetic mechanisms are involved in telomerase gene regulation in the hM SC. The chromatin-based repression of hTERT transcription and hence repression of telomerase activity may act as a tumor suppressor mechanism, whereby hM SC s would have a limited lifespan and can- not divide long enough to accumulate neoplastic changes and become tumorigenic. Basal transcription machinery dynamics were also changed upon TSA treatment. Association of Pol II with gene-coding regions has been previously shown to coincide with active gene transcription [23,24], and this agrees well with the data for hTERT for which we see an increased level of Pol II at exon 12 after treatment with TSA. Also apparent is a significant level of Pol II at the promoter sequence before treatment with TSA when hTERT is repressed. This may be due to a paused Pol II molecule being present at the promoter, perhaps indicating that the hTERT gene may be permissive for transcription, only needing changes in the 
Figure 2. hTR expression, chromatin environment and basal transcriptional machinery dynamics.
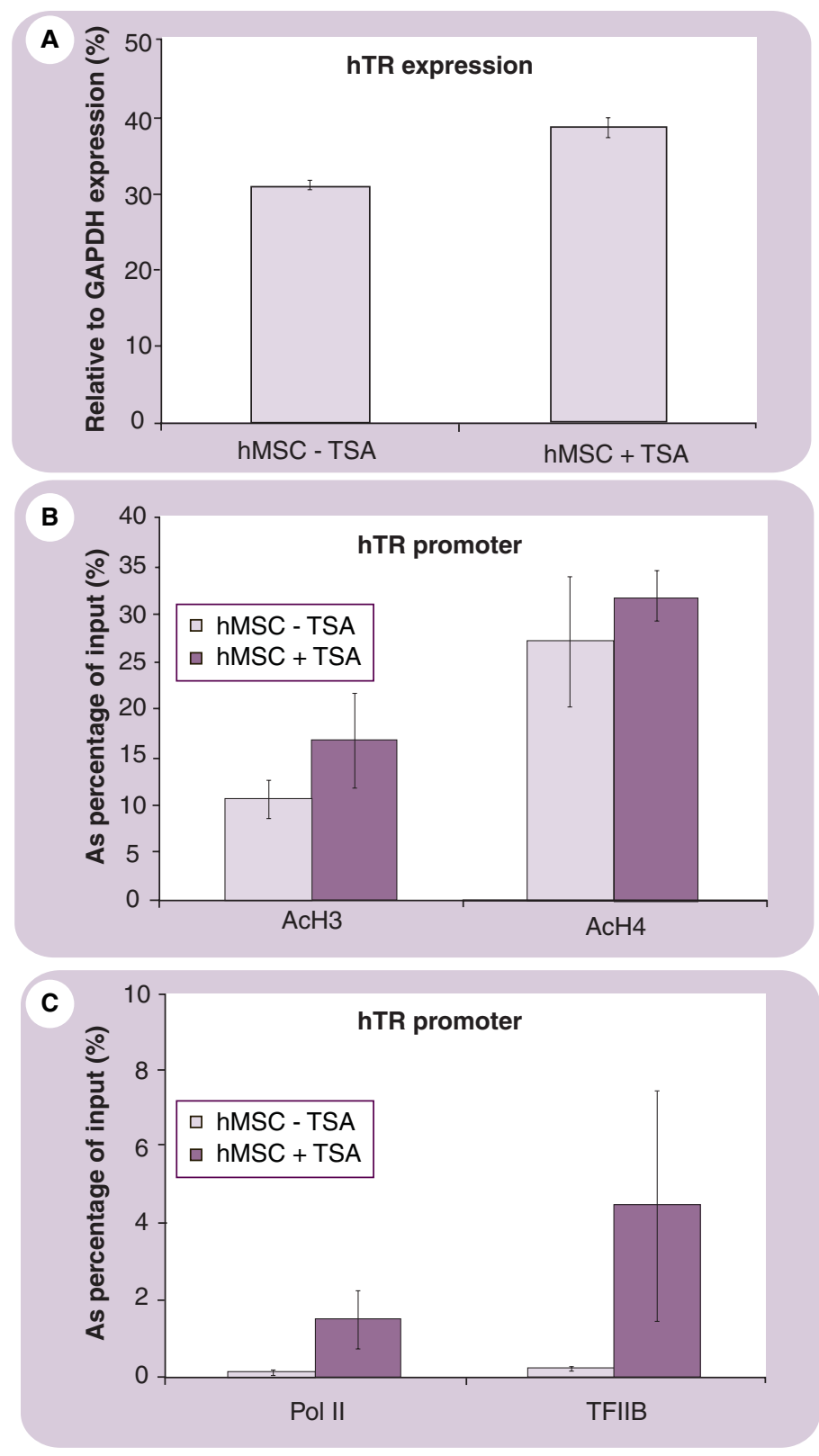

(A) hTR expression analysis for hM SCs before and after treatment with TSA, detected by Q-PCR and corrected to GAPDH expression. ChIP assays were performed for (B) histone acetylation status (histones $\mathrm{H} 3$ and H4) of the hTR promoter and (C) Pol II and TFIIB occupancy of the hTR promoter. Q-PCR: Quantitative polymerase chain reaction; TSA: Trichostatin A. chromatin environment of the promoter to allow transcription (Figure3). The apparent decrease in Pol II binding at the promoter and the large increase at exon 12 can be accounted for by increased Pol II tracking along the gene, being no longer paused at the promoter.
Apparent differences in Pol II dynamics between hT R and hTERT may reflect the different regulation of each of these genes. $C$ hanges in the chromatin environment of promoter sequences may therefore drive changes in the dynamics of the basal transcription machinery and complex interactions between transcriptional complexes and chromatin may therefore be important in mediating transcriptional activity.

While TSA treatment does lead to an increase in $h T E R T$ and $h T R$ expression when compared with the telomerase-positive M CF7 breast carcinoma cell line (Figure1A) hTERT expression in the hM SC treated with TSA is very low. It has also been observed that these cells display no detectable telomerase activity as measured by telomerase repeat amplification protocol (TRAP) [DATA NOT INCLUDED]. This observation indicates that while modulation of the chromatin environment by TSA is important in the control of hTERT and hTR expression, this action alone is not enough to increase levels of hTERT and hTR expression to a level high enough to reconstitute telomerase activity. This may be due to the lack of other factors that can influence gene expression.

It is known that hyperacetylation of promoter-specific histones at promoter sequences will cause relaxation of chromatin environment relative to a compacted state when histones are hypoacetylated. This relaxation of the chromatin environment will allow the binding of transcription factors, basal transcription machinery and other chromatin modifying enzymes to the promoter DNA. However, if such factors are not freely available within the cell, the relaxation of the chromatin environment alone will not be sufficient to mediate high levels of transcription. Analysis of the binding of regulatory factors influencing $h T E R T$ and $h T R$ expression in the hM SC will therefore be an important next step. Further analysis of histone modifications involved in telomerase gene repression in the hM SC may allow the identification of enzymes and co-regulators involved, which can then be targeted to allow a more specific method of modulating telomerase gene expression other than TSA.

Although TSA treatment has been shown to cause chromatin changes at the hTERT and hT R promoter, it is not known if this is a direct action. H owever, the use of TSA does allow us to see that changes in the chromatin environment, be they direct or indirect, can lead to changes in telomerase gene expression. These data provide 


\section{Figure 3. Chromatin and basal transcriptional machinery dynamics in hMSCs.}

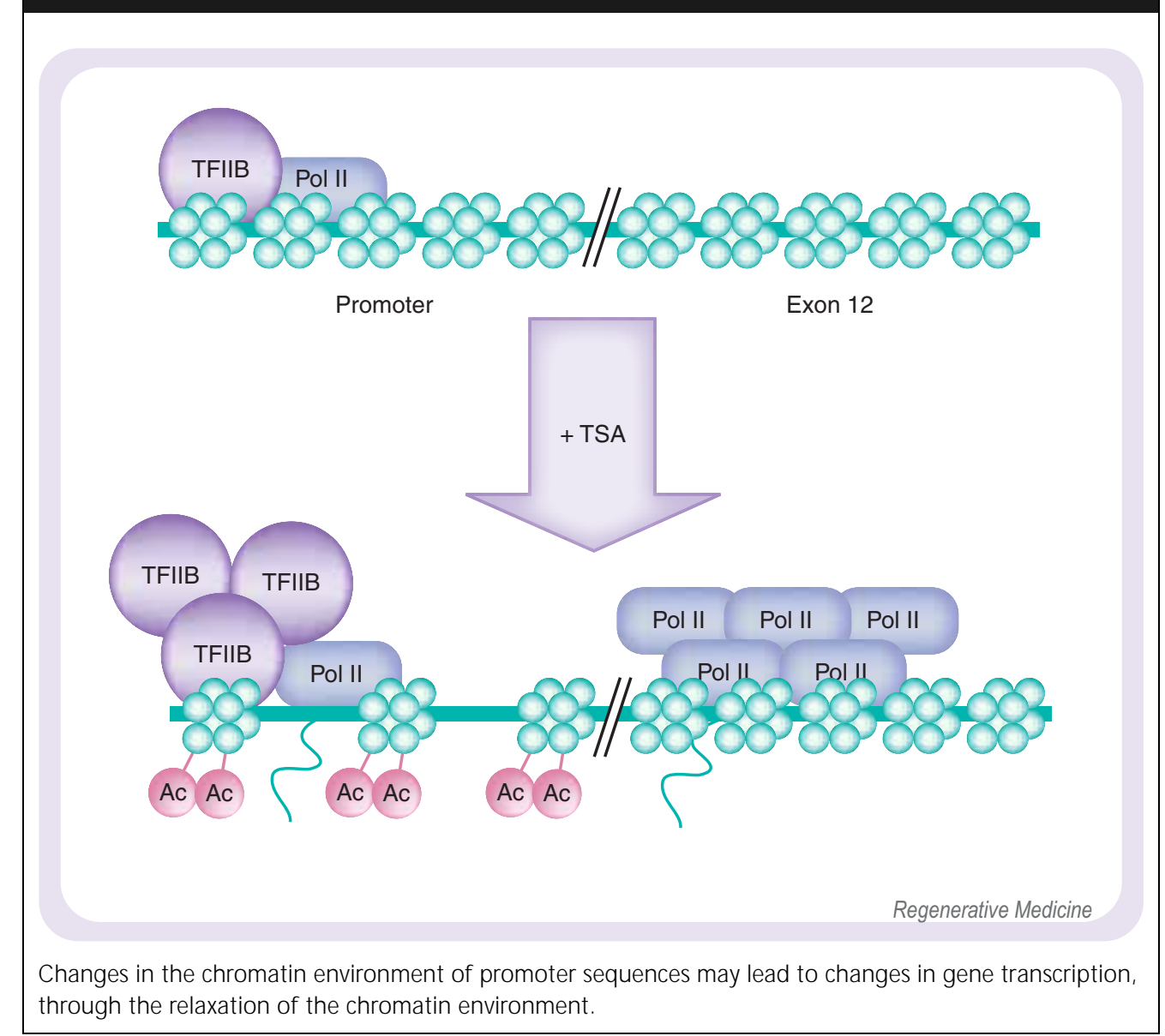

an impetus for further, more detailed, analysis of the interplay between the chromatin environment, general transcription machinery and other

\section{Executive summary}

- Human mesenchymal stem cells (hMSC) have limited replicative capacity and lack expression of the immortalizing enzyme telomerase.

- Lack of telomerase may act as a tumour suppressor mechanism as ectopic expression of telomerase in hMSC results in neoplastic transformation and reveals a cancer stem cell potential.

- Lack of telomerase gene expression is due to the repressive chromatin environment surrounding the telomerase gene promoters.

- Activation of telomerase gene expression is accompanied by chromatin remodelling and dynamic shifts in the basal transcriptional complex.

- hMSC represent an excellent model for studying telomerase gene expression with direct implications for cell replacement therapies and tissue engineering. important regulatory factors which may affect telomerase gene expression.

$O$ verall, this study shows that the lack of hT ERT expression in $\mathrm{hM} \mathrm{SC}$ s is due to a repressive chromatin environment surrounding the hT ERT gene promoter and that modulation of the chromatin environment has an important role in the reexpression of hTERT in these cells. This provides an excellent platform for the further investigation of the control of telomerase gene expression in the hM SC and its implications for tissue regeneration and cancer stem cells.

Acknowledgements

This work was supported by Cancer Research UK, European Community grants FIGH-CT-2002-00217, LSHC-CT2004-502943, Glasgow U niversity and D anish C ancer Society. 


\section{Chromatin and telomerase gene expression - RESEARCH ARTICLE}

\section{Bibliography}

1. Zimmermann S, Voss M , Kaiser S et al.: Lack of telomerase activity in human mesenchymal stem cells. Leukemia 17, 1146-1149 (2003).

2. Simonsen JL, Rosada C, Serakinci N et al.: Telomerase expression extends the proliferative lifespan and maintains the osteogenic potential of human bone marrow stromal cells. Nat. Biotechnol. 20, 592-596 (2002).

3. Dick JE: Breast cancer stem cells revealed. Proc. N atl Acad. Sci. USA 100, 3547-3549 (2003).

4. Serakinci N, Guldberg P, BurnsJS et al.: Adult human mesenchymal stem cell as a target for neoplastic transformation. Oncogene 23, 5095-5098 (2004).

5. Shay JW, Bacchetti S: A survey of telomerase activity in human cancer. Eur. J. Cancer 33, 787-791 (1997).

6. Kim N W, Piatyszek M A, Prowse KR et al.: Specific association of human telomerase activity with immortal cells and cancer. Science 266, 2011-2015 (1994).

7. H olt SE, Shay JW: Role of telomerase in cellular proliferation and cancer. J. Cell. Physiol. 180, 10-18 (1999).

8. Keith W N, Bilsland A, EvansT RJ, Rosalind $M$ : Telomerase-directed molecular therapeutics. Expert Rev. M ol. M ed. www.expertreviews.org/02004507h.htm (2002).

9. H orikawa I, Barrett JC: Transcriptional regulation of the telomerase hTERT gene as a target for cellular and viral oncogenic mechanisms. Carcinogenesis 24, 1167-1176 (2003).

10. Strahl $B D$, Allis $C D$ : The language of covalent histone modifications. $N$ ature 403 , 41-45 (2000).

11. Narita $\mathrm{M}, \mathrm{N}$ unez $\mathrm{S}, \mathrm{H}$ eard $\mathrm{E}$ et al.: $\mathrm{Rb}$ mediated heterochromatin formation and silencing of $\mathrm{E} 2 \mathrm{~F}$ target genes during cellular senescence. Cell 113, 703-716 (2003).

12. Bachman KE, Park BH, Rheel et al.: $H$ istonemodifications and silencing prior to D N A methylation of a tumor suppressor gene. Cancer Cell 3, 89-95 (2003).

13. Fisher AG. C ellular identity and lineage choice. N at. Rev. Immunol. 2, 977-982 (2002).

14. Atkinson SP, H oare SF, Glasspool RM et al.: Lack of telomerase gene expression in alternative lengthening of telomere cells is associated with chromatin remodeling of the hTR and hTERT gene promoters. Cancer Res. 65, 7585-7590 (2005).

15. Anderson $C J, H$ oare SF, Ashcroft $M$, Bilsland $A E$, K eith $W N$ : $H$ ypoxic regulation of telomerase gene expression by transcriptional and post-transcriptional mechanisms. Oncogene doi:10.1038/sj.onc.1209011. Epub ahead of print (2005).

16. Cong YS, Bacchetti $\mathrm{S}: \mathrm{H}$ istone deacetylation is involved in the transcriptional repression of hTERT in normal human cells. J. Biol. Chem. 275, 35665-35668 (2000).
17. Hou M, Wang X, Popov N et al.: The histone deacetylase inhibitor trichostatin $\mathrm{A}$ derepresses the telomerase reverse transcriptase (hTERT) gene in human cells. Exp. Cell Res. 274, 25-34 (2002).

18. Takakura M, Kyo S, Sowa $Y$ et al.: Telomerase activation by histone deacetylase inhibitor in normal cells. N ucleic Acids Res. 29, 3006-3011 (2001).

19. Pittenger M F, M ackay AM , Beck SC et al.: Multilineage potential of adult human mesenchymal stem cells. Science 284, 143-147 (1999).

20. Gomez-Roman N, Grandori C, Eisenman $\mathrm{RN}$ et al.: Direct activation of RNA polymerase III transcription by C-M yc. N ature 421, 290-294 (2003).

21. Keith W N , H oare SF : D etection of telomerase hTERT gene expression and its splice variants by RT-PCR. M ethods $M$ ol. M ed. 97, 297-309 (2004).

22. $H$ ahn W C, Weinberg RA: M odelling the molecular circuitry of cancer. $\mathrm{N}$ at. Rev. Cancer 2, 331-341 (2002).

23. Sandoval J, Rodriguez J L, Tur $G$ et al.: RN APol-ChIP: a novel application of chromatin immunoprecipitation to the analysis of real-time gene transcription. N ucleic Acids Res. 32, e88 (2004).

24. Zhang $L$, Johnson $M$, Le KH et al.: Interrogating androgen receptor function in recurrent prostate cancer. Cancer Res. 63, 4552-4560 (2003). 